\title{
BMJ Open Organisational interventions designed to reduce caesarean section rates: a systematic review protocol
}

\author{
Alison M Hutchinson, ${ }^{1,2}$ Cate Nagle, ${ }^{3,4}$ Bridie Kent, ${ }^{5}$ Debra Bick, ${ }^{6}$ \\ Rebecca Lindberg ${ }^{7}$
}

To cite: Hutchinson AM, Nagle C, Kent B, et al. Organisational interventions designed to reduce caesarean section rates: a systematic review protocol. BMJ Open 2018;8:e021120. doi:10.1136/ bmjopen-2017-021120

- Prepublication history for this paper is available online. To view these files please visit the journal online (http://dx.doi org/10.1136/bmjopen-2017021120).

Received 13 December 2017 Revised 10 March 2018 Accepted 1 June 2018
D) Check for updates

(c) Author(s) (or their employer(s)) 2018. Re-use permitted under CC BY-NC. No commercial re-use. See rights and permissions. Published by BMJ.

For numbered affiliations see end of article.

\section{Correspondence to} Dr Alison M Hutchinson; alison.hutchinson@deakin. edu.au

\section{ABSTRACT}

Introduction There is a growing body of evidence to indicate that both primary and subsequent caesarean sections are associated with increased maternal and perinatal morbidity. Efforts to reduce the number of clinically unnecessary caesarean sections are urgently required. Our objective is to systematically review published evidence on the effectiveness of maternity service organisational interventions, such as models of maternity care, that aim to reduce caesarean section rates. Methods and analysis Databases will be searched, including the Cochrane Central Register of Controlled Trials, Cumulative Index to Nursing and Allied Health Literature, MEDLINE, Maternity and Infant Care, EMBASE and SCOPUS. Search terms related to caesarean section and organisational intervention will be used. Research published before 1980 will be excluded and only randomised controlled trials, cluster-randomised controlled trials, quasi-randomised controlled trials, controlled before and after studies and interrupted time series studies will be included. Data extraction and quality assessments will be undertaken by two authors.

Ethics and dissemination Ethics approval is not required for this systematic review. The results of this study will be disseminated via peer-reviewed publication and presentation at professional conferences. PROSPERO registration number CRD42016039458.

\section{INTRODUCTION}

Based on systematic review evidence, the WHO recently concluded that caesarean section rates of greater than $10 \%$ are not linked to lower maternal and neonatal mortality rates. ${ }^{12}$ In 2014, the rates of childbirth by caesarean section across 150 countries ranged from $6 \%$ to $27.2 \%$ (average $18.6 \%) .{ }^{3}$ In high-income countries, the use of caesarean sections has increased steadily over the last decade. ${ }^{3}$ The reasons for the increase are varied and complex and are reported to include malpractice liability, an increase in acuity of women birthing, scheduling convenience and an increase in the number of women with pre-existing medical complications, such as diabetes, when they become pregnant. $^{3-5}$ Professional associations in

\section{Strengths and limitations of this study}

- The study protocol is informed by the Cochrane Collaboration systematic review methods and adheres to the Preferred Reporting Items for Systematic Reviews and Meta-Analyses Protocols.

Of the studies identified for inclusion, the full publication will be independently assessed for quality by two authors.

- The concept of an organisational intervention is not definitive and will likely require discussion among the research team to establish which interventions are included/excluded in the review.

- Only papers where caesarean section is the primary outcome measure will be included, hence potentially relevant studies may be omitted if caesarean section is a secondary outcome.

- Inadequate reporting of complex interventions used in individual studies may limit the usability of the review findings.

nations such as Canada, ${ }^{6}$ Australia and New Zealand $^{7}$ and the USA ${ }^{8}$ have issued guidelines to promote vaginal birth for uncomplicated pregnancies, whenever possible. It is also acknowledged in the guidelines that, based on individual assessment and need, planned caesarean sections should be recommended in selected cases, for example, if women have experienced previous (physical or psychological) trauma. ${ }^{9}$

While caesarean section expedites birth in obstetric emergencies, the role of planned surgical birth in reducing or minimising potential for subsequent morbidity, for example, providing perineal protection for a woman with previous severe perineal trauma, is as yet unknown. ${ }^{10}$ Planned and unplanned caesarean births are associated with adverse maternal and neonatal outcomes. Caesarean births increase the risk of maternal mortality compared with vaginal birth and significantly increase the risk of death through complications of anaesthesia, wound and genital tract infection and thromboembolism. ${ }^{11}$ 
Postoperative surgical wound infections have been reported to develop in around 5\% of women while still hospital inpatients. ${ }^{12}$ Genital tract sepsis was one of the leading causes of direct maternal death in women giving birth in the UK during 2009-2013, ${ }^{13}$ with most deaths occurring following discharge from hospital and all forms of operative birth associated with increased risk of sepsis. Planned caesarean section is associated with a higher risk of hysterectomy, anaesthetic complications and obstetric shock compared with planned vaginal birth. ${ }^{14}$ Increases in maternal morbidity occur with subsequent caesarean sections, including increased risks of placenta acreta, cystotomy, bowel injury, ureteral injury, ileus, postpartum ventilation, intensive care admission, haemorrhage, hysterectomy and blood transfusion. ${ }^{15}$ Other adverse maternal risks associated with caesarean sections are endometritis, thrombosis and bladder or ureter damage requiring further surgery. ${ }^{16}$ There are also worse health outcomes for women in subsequent pregnancies ${ }^{17}$; for example, the risk of peripartum hysterectomy increases if a woman has had a previous caesarean birth. ${ }^{18}$

Newborns delivered via caesarean section tend to have higher Apgar scores than those delivered vaginally, but require more oxygen resuscitation and have higher risk of onset of physiological jaundice compared with babies born vaginally. ${ }^{19}$ The duration of inpatient stay has traditionally been longer for women following caesarean section births compared with normal or operative vaginal births. For example, in Victoria, Australia, the 20092010 length of stay following caesarean section was 4.51 days compared with an inpatient duration of 1.93 days following vaginal birth. ${ }^{20}$ However, inpatient duration has been declining following caesarean births with anecdotal evidence that women in the UK are being discharged home within 48 hours of a planned caesarean birth following the introduction of enhanced recovery after surgery pathways for major elective surgery. ${ }^{21}$ The impact of early postnatal discharge following caesarean birth on women's health and other postnatal care outcomes in community settings has yet to be established.

With respect to neonatal outcomes, compared with planned vaginal births, babies born by elective caesarean section experience more admissions to neonatal units, greater risk of respiratory morbidity and higher mortality rates. ${ }^{22}$ Lower rates of breastfeeding initiation have been reported following caesarean birth as a consequence of a lack of effective pain relief impacting on a woman's ability to care for her baby, ${ }^{23}$ or due to separation of the mother and baby following birth. Surgery may impact on postpartum prolactin levels resulting in a subsequent delay in the onset of lactation, ${ }^{24}{ }^{25}$ which maternity staff may fail to address or take into account when planning care for women postoperatively. Furthermore, evidence suggests that caesarean section surgery is more costly than vaginal delivery in low-risk populations. ${ }^{26}$

Urgent calls have been made by organisations, for example, the US Office of Disease Prevention and Health Promotion ${ }^{27}$ and the $\mathrm{WHO},{ }^{28}$ to reduce the rates of unnecessary caesarean sections. Extrapolating from the complications associated with caesarean section, predicted outcomes from a reduction in caesarean section rates include: improved safety through reduced incidents and admissions of babies to neonatal care; reduced perinatal and maternal morbidity and mortality; higher rates of breastfeeding (particularly in the long term); improved quality of maternity care; significant cost savings and improved national compliance with WHO recommendations.

The study described in this protocol broadly seeks to establish evidence for the effectiveness of maternity service organisational interventions developed to reduce planned and unplanned caesarean births. The Cochrane Effective Practice and Organisation of Care group defines organisational interventions as '...those which involve a change in the structure or delivery of healthcare. In other words, an organisational intervention is a change in who delivers healthcare, how care is organised, or where care is delivered'. ${ }^{29}$

\section{METHODS AND ANALYSIS \\ Aim}

Our specific aim is to systematically review published evidence on maternity service organisational interventions that aim to reduce planned and unplanned caesarean section rates.

\section{Design}

This systematic review protocol is informed by the Cochrane Collaboration systematic review methods and adheres to the Preferred Reporting Items for Systematic Reviews and Meta-Analyses Protocols (PRISMA-P) ${ }^{30}$

The following criteria will be used to screen studies, based on the 'PICO' (participants, intervention, comparator and outcome) approach:

\section{Inclusion criteria}

Studies will be included only if they are published in peer-reviewed journals and report on organisational interventions and planned and unplanned caesarean section rates. Other inclusion criteria consist of:

- Study designs: randomised controlled trials (RCTs), cluster-randomised controlled trials, quasi-randomised controlled trials, controlled before and after studies and interrupted time series studies.

- Types of setting and participants: obstetric-led maternity services able to provide support for women undergoing planned or unplanned CS birth. Maternity care clinicians, including midwives, obstetricians, nurses, paediatricians, family doctors and anaesthetists, maternity care managers and maternity care educators.

- Types of interventions and comparators: These may take the form of maternity service organisational interventions, for example, feedback mechanisms, incentives, education, reminder mechanism and 
models of care. The strategy may comprise a single component (eg, education only) or be multifaceted (comprising two or more components, eg, education and reminders) aimed to reduce caesarean section rates versus usual care (no such organisational intervention). Some interventions, typically those that are multifaceted, comprising interacting components, are described as complex interventions. ${ }^{31}$

- Types of outcome measures: Studies that report objectively measured or self-reported (using validated instruments) outcomes. Planned, unplanned and overall caesarean section rates are expected to be the primary outcome measure. Secondary outcome measures include admissions to neonatal intensive care units or special care units, maternal adverse events (eg, postpartum haemorrhage, wound infection and thromboembolism), breastfeeding initiation and duration, maternal and newborn duration of inpatient stay, women's experiences of care, adherence to best practice guidelines by health professionals, health professionals' satisfaction, confidence, competence, attitudes, knowledge and self-efficacy.

We will not apply search restrictions according to language or the income level of countries. Based on title and abstract, potentially relevant publications in other languages will be translated into English by accredited professional translators.

\section{Exclusion criteria}

Exclusions will be made based on types of interventions. For the purposes of this study, medical interventions (eg, drugs to induce labour, episiotomy and instrumental delivery), lifestyle interventions (eg, nutrition and physical activity programmes for expectant mothers) and labour interventions (eg, water births, epidural analgesia, augmentation of labour) are not defined as organisational interventions and, hence, will be excluded.

\section{Setting}

The organisations relevant to this study include hospitals, maternity care clinics and health centres (described as maternity services). Organisational interventions could include, for example, staff training, service-wide policies or new models or methods of organising maternity care and decision support tools for women and clinicians.

\section{Search strategy}

The systematic search of the literature will involve three stages:

\section{Database search}

Studies published from 1 January 1980 to 31 December 2017 will be identified using the following databases:

- The Cochrane Central Register of Controlled Trials

- Cumulative Index to Nursing and Allied Health Literature

- MEDLINE

- Maternity and Infant Care

- EMBASE

\section{- SCOPUS}

Search terms will be developed from our knowledge of existing literature and advice of qualified health librarians, and will include controlled vocabulary and keyword terms related to: (1) caesarean (cesarean, caesarian, cesarian) section AND (2) organis(z)ational interventions AND (3) quality outcomes AND (4) research design. The search terms will be applied according to the nuances of each database; as an example, see the planned MEDLINE search in box 1 . The platforms for each database are outlined in table 1 .

\section{Systematic literature reviews}

Published systematic literature reviews, captured in our database search and identified as relevant to our review objective, will be used to cross-check included studies and identify additional eligible references.

\section{Reviewing reference lists}

The reference lists of included studies will be reviewed to identify additional eligible references.

\section{Screening}

EndNote X7 will be used to manage the results of all searches and to facilitate the screening process. A unique identifier will be automatically assigned by EndNote X7 to every publication retrieved in the three-stage search process to enable tracking of articles. Following deduplication, two authors will independently screen the titles and abstracts of all publications retrieved and an interrater calculation will be performed to reveal the level of agreement. A Microsoft Excel spreadsheet will be used to track the screening process.

Of the studies identified for inclusion, the full publications will be retrieved and reviewed by two authors. Following review of the full text, the inclusion/exclusion status and rationale will be recorded for each study. All disagreements regarding inclusion/exclusion will be resolved through consensus. The PRISMA ${ }^{30}$ flow diagram will be used for reporting the screening process.

\section{Data extraction}

A purpose-designed Microsoft Excel spreadsheet will be used for recording extracted data. One author will independently extract data from each included publication. Extracted data will include: year, authors, publication title, research question or study purpose, study design, context, subjects/participants, sample size, theoretical/ conceptual framework, intervention (type, elements), definition of concepts, data collection methods, instrumentation, reliability coefficients, validity measures and relevant results (the relationship between caesarean section rates and organisational interventions). Extracted data will be checked for accuracy and completeness by a second author and any discrepancies resolved by consensus. 


\section{Box 1 Search terms used in MEDLINE}

1. Cesarean Section/

2. ((caesarean or cesarean) adj (section? or birth? or deliver\$)).ti,ab.

3. (c-section? or c section).ti,ab.

4. ((Peri natal or peri-natal) adj care).ti,ab.

5. or $/ 1-4$

6. *health maintenance organizations/

7. exp Organizational Innovation/

8. exp Health Services/

9. Health Facilities/

10. 9

11. exp Hospitals/

12. exp Hospital Restructuring/

13. exp Health Planning/

14. Health Policy/

15. Clinical Governance/

16. exp Maternal Health Services/

17. exp 'Organization and Administration'/

18. Program Development/

19. Capacity Building/

20. exp 'Delivery of Health Care'/

21. 'Attitude of Health Personnel'/

22. exp 'Quality of Health Care'/

23. exp 'Risk Management'/

24. adverse event.ti,ab.

25. exp Patient Care Team/

26. interdisciplinary.ti,ab.

27. exp 'Interprofessional Relations'/

28. exp 'Quality Assurance, Health Care'/

29. exp Motivation/

30. exp safety/or patient safety/

31. organizational culture/

32. ((program* or facilit*) adj1 (health or care or intervention $\left.{ }^{\star}\right)$ ).ti,ab.

33. capacity building.ti,ab.

34. (organi ${ }^{\star}$ adj2 (learning or change or changing or management or restructure or redesign or develop*)).ti,ab.

35. ((quality or safety or change) adj2 (program or improve $\left.{ }^{\star}\right)$ ).ti,ab.

36. or/6-35

37. and $/ 5,36$

38. exp Morbidity/

39. exp Mortality/

40. exp risk/

41. ae.fs.

42. co.fs.

43. audit*.ti,ab.

44. or $/ 38-43$

45. 37 and 44

46. randomized controlled trial.pt.

47. controlled clinical trial.pt.

48. randomized.ab.

49. placebo.ab.

50. drug therapy.fs.

51. randomly.ab.

52. trial.ab.

53. groups.ab.

54. or/46-53

55. exp animals/nothumans.sh.

56. 54 not 55

57. and $/ 45,56$

58. limit 57 to $\mathrm{yr}=$ '1980-Current'
Table 1 Platforms for each database

\begin{tabular}{ll} 
Database & Platform \\
\hline $\begin{array}{l}\text { The Cochrane Central Register of } \\
\text { Controlled Trials }\end{array}$ & $\begin{array}{l}\text { The Cochrane Library } \\
\text { through Wiley Online } \\
\text { Library }\end{array}$ \\
$\begin{array}{l}\text { Cumulative Index to Nursing and } \\
\begin{array}{l}\text { Allied Health Literature (CINAHL) } \\
\text { (excluding MEDLINE) }\end{array}\end{array}$ & EBSCO \\
MEDLINE & Ovid \\
Maternity and Infant Care & Ovid \\
EMBASE (excluding MEDLINE) & EMBASE \\
SCOPUS & Sciverse \\
\hline
\end{tabular}

\section{Methodological quality assessments}

Each included publication will be independently assessed for quality by two authors. The risk of bias tool recommended by the Cochrane Collaboration and described in the Cochrane Handbook for Systematic Reviews of Interventions ${ }^{32}$ will be used for RCTs. This tool addresses six domains: sequence generation; allocation concealment; blinding of participants, personnel and outcome assessors; incomplete outcome data; selective outcome reporting; and other potential threats to validity. ${ }^{32}$ Each domain will be graded as either 'low risk', 'high risk' or 'unclear risk' for bias.

The Quality Assessment Tool for Quantitative Studies ${ }^{33}$ will be used for all other research designs. This tool addresses eight domains: selection bias, study design, confounders, blinding, data collection, participant withdrawals, intervention integrity and analysis. The quality assessment across the eight domains allows an overall quality rating to be achieved: 'strong', 'moderate' or 'weak'. This tool has been evaluated for construct and content validity and was found to have excellent interrater agreement for the overall quality rating. ${ }^{34}$

Discrepancies in author ratings using either tool will be identified, discussed and resolved by consensus. Risk of bias and quality rating will be considered in the analysis of data (sensitivity analysis), but will not determine a study's inclusion or exclusion. For evidence of potential reporting biases, funnel plots will be used.

\section{Data analysis and synthesis}

Measures of treatment effect

Where studies report dichotomous outcomes, we will report results as a summary risk ratio with $95 \%$ CI. For studies reporting continuous outcomes, we will report the mean difference if outcomes are measured in the same way between studies. We will use the standardised mean difference to combine trials that measure the same outcome, but use different methods.

For controlled before and after studies, we will report relative effects. For dichotomous outcomes, we will report the risk ratio adjusting for baseline differences in the outcome measures. For continuous variables, we 
will report on the relative change, adjusting for baseline differences in the outcome measures.

For interrupted time series studies, the changes in intercept and slope coefficients will be compared between the postintervention and preintervention periods via segmented regression technique, controlling for the overall rate of outcome trend. Mean changes will be used to combine studies measuring the same outcomes.

\section{Cluster-randomised trials}

We will combine results from cluster-randomised trials with individually randomised trials if there is little heterogeneity between the study designs and the interaction between the effect of intervention and the choice of randomisation unit is considered to be unlikely. We will adjust their SE using the reported intracluster correlation coefficient. We will also acknowledge heterogeneity in the unit of randomisation and perform a sensitivity analysis to investigate the effects of the randomisation unit.

\section{Subgroup analysis}

Subgroup analyses, based on the Robson caesarean section classification system, will be undertaken to assess the effectiveness of interventions by participant type. The 10-Group Classification System was developed to enable analysis of events and outcomes associated with labour and delivery according to different types of clinical intervention. $^{35}$

\section{Dealing with missing data}

For included studies, we will note levels of attrition. A sensitivity analysis will be undertaken to assess the impact of including studies with high levels of missing data in the overall assessment of treatment effect. For all outcomes, we will carry out analyses, as far as possible, on an intention-to-treat basis. The denominator for each outcome in each trial will be the number randomised minus any participants whose outcomes are known to be missing.

\section{Assessment of heterogeneity}

We will assess statistical heterogeneity in each meta-analysis using the $\tau^{2}, \mathrm{I}^{2}$ and $\chi^{2}$ statistics. We will regard heterogeneity as substantial if an $\mathrm{I}^{2}$ is greater than $30 \%$, and either the $\tau^{2}$ is greater than zero or there is a low $\mathrm{P}$ value (less than 0.10 ) in the $\chi^{2}$ test for heterogeneity.

\section{Data synthesis}

We will conduct statistical analysis using the Review Manager software. ${ }^{36}$ A fixed-effect meta-analysis with $95 \%$ CI will be used for combining data where it is reasonable to assume that studies are estimating the same intervention, and the study populations and methods are assessed to be similar. If significant heterogeneity is detected, we will use random-effects meta-analysis to produce an overall summary. If we use random-effects analyses, the results will be presented as the average treatment effect with $95 \% \mathrm{CI}$, and the estimates of $\tau^{2}$ and $\mathrm{I}^{2}$.

In the event that studies have significant clinical heterogeneity, we will present narrative, descriptive and qualitative reporting of the data. Because of the complex nature of, and variation in, organisational interventions, there may be inadequate numbers of studies that are similar enough to enable combination of the results statistically. The approach for the narrative reporting will be informed by guidelines described by Grimshaw. ${ }^{37}$ This will include: first, grouping studies into categories based on the style of the organisational intervention, research design and setting; then, conducting within study analysis to describe the findings and characteristics, quality of the study and design; and finally, cross-study synthesis to aggregate all included studies, compare the effectiveness of different interventions and discuss the relationships in the findings.

\section{Sensitivity analysis}

We will perform sensitivity analysis based on the risk of bias in the randomisation, allocation concealment, blinding and loss to follow-up of the studies.

\section{Protocol amendments}

Any amendments to the protocol will be documented on the International Prospective Register of Systematic Reviews (PROSPERO) and in the final manuscript.

\section{Patient and public involvement}

Patients and the public were not involved in the development of this protocol and they will not participate in the conduct of the systematic review.

\section{DISCUSSION}

This paper outlines a protocol for the systematic review search strategy, extraction method, synthesis and appraisal of the quality of published evidence in relation to maternity service organisational interventions that aim to reduce planned and unplanned caesarean section rates. The results of this study will provide a framework to assist decision makers in maternity service settings regarding the anticipated outcomes of, and most effective approaches for, reducing caesarean rates. The results will address a gap in the literature and help to answer calls for reduced caesarean section rates globally.

As described in this protocol, the Cochrane Collaboration's guide for systematic reviews of the literature is used to inform the research design, with three main adaptions. First, screening of studies included in previously published systematic reviews that addressed a similar but not identical question, will be undertaken. This will improve the rigour of our search strategy and enable a cross-check of our database search yield. Second, it is typical to use strict criteria when screening studies. In our protocol, we have added detail at every level of the inclusion criteria (design, participants, intervention type and outcome measures) and exclusion criteria (describing excluded interventions) to expedite the screening process and provide clear criteria for reviewers. Finally, two quality assessment tools will be used in our research. 
Although risk of bias tools are appropriate for RCTs, because we anticipate including other research designs, a second quality assessment tool is included to enable assessment of other study designs.

This protocol has potential study design limitations. The definition of an organisational intervention is not definitive and, hence, we anticipate that including/ excluding on these criteria will involve robust discussion between reviewers to achieve a consensus position. Relevant examples of organisational interventions for this setting will be revealed with potential application for future research and practice. The screening tool stipulates that we will also exclude papers where caesarean sections are not the primary outcome measure. Hence, interventions where caesarean sections are a secondary outcome will not be included and this may exclude potentially relevant studies. Risk of bias assessments have been found to be difficult to implement in terms of two domains: incomplete outcome data and selective reporting of outcomes ${ }^{38}$ and this may limit the validity of assessments performed. To reduce the likelihood of these domains impacting our assessments, two authors will perform risk of bias assessments for all included studies, discuss discrepancies in decisions and draw on the Cochrane Collaboration's supporting material as required. In the event that consensus is not reached, a third author will be consulted for a final decision. Finally, inconsistent and insufficient detail in the reports of individual studies of complex interventions may limit the usefulness of the review findings.

In conclusion, the reported outcomes of maternity service organisational interventions that aim to reduce caesarean section rates will be synthesised and statistically (if possible) and narratively compared. The results will be useful in informing decisions about optimal organisational approaches to reduce the rates of caesarean sections in maternity settings. Predicted benefits arising from a reduction in rates include improved maternal and infant care and cost-savings for resource-stretched healthcare providers

\author{
Author affiliations \\ ${ }^{1}$ School of Nursing and Midwifery, Centre for Quality and Patient Safety, Deakin \\ University, Geelong, Victoria, Australia \\ ${ }^{2}$ Deakin Centre for Quality and Patient Safety Research, Monash Health, Clayton, \\ Victoria, Australia \\ ${ }^{3}$ College of Healthcare Sciences, James Cook University, Townsville, Queensland, \\ Australia \\ ${ }^{4}$ Townsville Hospital and Health Service, Townsville, Queensland, Australia \\ ${ }^{5}$ Faculty of Health and Human Sciences, University of Plymouth, Plymouth, Devon, \\ UK \\ ${ }^{6}$ Department of Women and Children's Health, School of Life Course Sciences, \\ Faculty of Life Sciences and Medicine, Kings College London, London, UK \\ ${ }^{7}$ Institute for Physical Activity and Nutrition, School of Exercise and Nutrition \\ Sciences, Deakin University, Geelong, Victoria, Australia
}

Acknowledgements The authors acknowledge the contributions of Justin Calache as Research Assistant and Deakin University research librarians, Louisa Sher and Rachel West.

Contributors $\mathrm{AMH}, \mathrm{CN}$ and $\mathrm{BK}$ conceived of the original research project. $\mathrm{AMH}$ and $\mathrm{CN}$ developed the protocol. AMH managed the first draft of this manuscript and coordinated the contributions of coauthors. BK and DB advised on the protocol design. CN, DB and BK commented on the draft manuscript and contributed to the content and edits. RL assisted with the development and execution of the search strategy and drafting and coordination of the manuscript. All authors have read and approved the final manuscript.

Funding The authors have not declared a specific grant for this research from any funding agency in the public, commercial or not-for-profit sectors.

Disclaimer The views expressed are those of the author(s) and not necessarily those of the NHS, the NIHR or the Department of Health and Social Care.

Competing interests DB is supported by the National Institute for Health Research (NIHR) Collaboration for Leadership in Applied Health Research and Care South London.

Patient consent Not required.

Provenance and peer review Not commissioned; externally peer reviewed.

Open access This is an open access article distributed in accordance with the Creative Commons Attribution Non Commercial (CC BY-NC 4.0) license, which permits others to distribute, remix, adapt, build upon this work non-commercially, and license their derivative works on different terms, provided the original work is properly cited, appropriate credit is given, any changes made indicated, and the use is non-commercial. See: http://creativecommons.org/licenses/by-nc/4.0/.

\section{REFERENCES}

1. World Health Organization. WHO Statement on Caesarean Section Rates. 2015 http://www.who.int/reproductivehealth/publications/ maternal_perinatal_health/cs-statement/en/ (accessed 5 Feb 2018).

2. Betran AP, Torloni MR, Zhang JJ, et al. WHO statement on caesarean section rates. BJOG 2016;123:667-70.

3. Betrán AP, Ye J, Moller AB, et al. The increasing trend in caesarean section rates: Global, regional and national estimates: 1990-2014. PLoS One 2016;11:e0148343.

4. Roos-Hesselink JW, Ruys TP, Stein JI, et al. Outcome of pregnancy in patients with structural or ischaemic heart disease: results of a registry of the European Society of Cardiology. Eur Heart $J$ 2013;34:657-65.

5. Scott-Pillai R, Spence D, Cardwell CR, et al. The impact of body mass index on maternal and neonatal outcomes: a retrospective study in a UK obstetric population, 2004-2011. BJOG 2013;120:932-9.

6. Society of Obstetricians and Gynaecologists of Canada. Joint Policy Statement on Normal Childbirth - Policy Statement. 2008 http:// www.jogc.com/article/S1701-2163(16)34029-4/pdf (accessed 21 Apr 2017).

7. The Royal Australian and New Zealand College of Obstetricians and Gynaecologists. Timing of Elective Caesarean Section at Term, 2012.

8. Caughey AB, Cahill AG, Guise J, et al. Safe prevention of the primary cesarean delivery. Obstetric Care Consensus Series. 2014 http://www.acog.org/Resources-And-Publications/Obstetric-CareConsensus-Series/Safe-Prevention-of-the-Primary-CesareanDelivery (accessed 21 Apr 2017).

9. Fernando RJ, Sultan AH, Freeman RM, et al. The Management of Third- and Fourth-Degree Perineal Tears. Green-top Guideline No. 29. 2015 https://www.rcog.org.uk/globalassets/documents/ guidelines/gtg-29.pdf (accessed 17 Feb 2018).

10. Farrar D, Tuffnell DJ, Ramage C. Interventions for women in subsequent pregnancies following obstetric anal sphincter injury to reduce the risk of recurrent injury and associated harms. Cochrane Database Syst Rev 2014:CD010374.

11. Kamilya G, Seal SL, Mukherji J, et al. Maternal mortality and cesarean delivery: an analytical observational study. J Obstet Gynaecol Res 2010;36:248-53.

12. Ward VP, Charlett A, Fagan J, et al. Enhanced surgical site infection surveillance following caesarean section: experience of a multicentre collaborative post-discharge system. J Hosp Infect 2008;70:166-73.

13. Knight M, Tuffnell D, Kenyon S, eds. Confidential Enquiries into Maternal Deaths and Morbidity 2009-13. Oxford, UK: National Perinatal Epidemiology Unit, University of Oxford, 2015.

14. Farchi S, Polo A, Franco F, et al. Severe postpartum morbidity and mode of delivery: a retrospective cohort study. Acta Obstet Gynecol Scand 2010;89:1600-3.

15. Silver RM, Landon MB, Rouse DJ, et al. Maternal morbidity associated with multiple repeat cesarean deliveries. Obstet Gynecol 2006;107:1226-32. 
16. Kulas T, Bursac D, Zegarac Z, et al. New Views on Cesarean Section, its Possible Complications and Long-Term Consequences for Children's Health. Med Arch 2013;67:460-3.

17. Kok N, Ruiter L, Hof M, et al. Risk of maternal and neonatal complications in subsequent pregnancy after planned caesarean section in a first birth, compared with emergency caesarean section: a nationwide comparative cohort study. BJOG 2014;121:216-23.

18. de la Cruz CZ, Thompson EL, O'Rourke K, et al. Cesarean section and the risk of emergency peripartum hysterectomy in highincome countries: a systematic review. Arch Gynecol Obstet 2015;292:1201-15

19. Geller EJ, Wu JM, Jannelli ML, et al. Neonatal outcomes associated with planned vaginal versus planned primary cesarean delivery. $J$ Perinatol 2010;30:258-64.

20. Department of Health. The Victorian Admitted Episode Dataset. The State Government of Victoria. 2011 http://www.health.vic.gov.au (accessed 28 Aug 2015).

21. Corso E, Hind D, Beever D, et al. Enhanced recovery after elective caesarean: a rapid review of clinical protocols, and an umbrella review of systematic reviews. BMC Pregnancy Childbirth 2017;17:91.

22. De Luca R, Boulvain M, Irion O, et al. Incidence of early neonatal mortality and morbidity after late-preterm and term cesarean delivery. Pediatrics 2009;123:e1064-e1071.

23. Zanardo V, Pigozzo A, Wainer G, et al. Early lactation failure and formula adoption after elective caesarean delivery: cohort study. Arch Dis Child Fetal Neonatal Ed 2013;98:F37-F41.

24. Wang BS, Zhou LF, Zhu LP, et al. [Prospective observational study on the effects of caesarean section on breastfeeding]. Zhonghua Fu Chan Ke Za Zhi 2006;41:246-8.

25. Scott JA, Binns CW, Oddy WH. Predictors of delayed onset of lactation. Matern Child Nutr 2007;3:186-93.

26. Petrou S, Khan K. An overview of the health economic implications of elective caesarean section. Appl Health Econ Health Policy 2013;11:561-76.

27. Office of Disease Prevention and Health Promotion. Healthy People 2020. Washington, DC: U.S: Office of Disease Prevention and Health Promotion, 2016. (accessed 7 Feb 2018).
28. World Health Organization. Caesarean sections should only be performed when medically necessary. News Release 10 Apri 2015 http://www.who.int/mediacentre/news/releases/2015/caesareansections/en/ (accessed 30 Sep 2015)

29. Effective Practice and Organisation of Care (EPOC). EPOC Taxonomy. $2015 \mathrm{https}$ ://epoc.cochrane.org/epoc-taxonomy (accessed 21 Apr 2017)

30. Moher D, Liberati A, Tetzlaff J, et al. Preferred reporting items for systematic reviews and meta-analyses: the PRISMA statement. PLoS Med 2009;6:e1000097.

31. Craig P, Dieppe P, Macintyre S, et al. Developing and evaluating complex interventions: the new Medical Research Council guidance. BMJ 2008;337:a1655

32. Cochrane Handbook for Systematic Reviews of Interventions Version 5.1.0. In: Higgins JPT, Green S, eds. The Cochrane Collaboration, 2011. (accessed 21 Apr 2017).

33. Effective Public Health Practice Project (EPHPP). Quality Assessment Tool for Quantitative Studies. Hamilton, Ontario, Canada: Effective Public Health Practice Project, 2010. http://www.ephpp.ca/PDF/ Quality\%20Assessment\%20Tool_2010_2.pdf (accessed 21 Apr 2017).

34. Armijo-Olivo S, Stiles CR, Hagen NA, et al. Assessment of study quality for systematic reviews: a comparison of the Cochrane Collaboration Risk of Bias Tool and the Effective Public Health Practice Project Quality Assessment Tool: methodological research. J Eval Clin Pract 2012;18:12-18.

35. Robson M, Murphy M, Byrne F. Quality assurance: the 10-Group Classification System (Robson classification), induction of labor, and cesarean delivery. Int J Gynaecol Obstet 2015;131(Suppl 1):S23-7.

36. Review Manager (RevMan) [Computer program]. Version 5.3. Copenhagen: The Nordic Cochrane Centre, The Cochrane Collaboration, 2014.

37. Grimshaw J. A Guide to Knowledge Synthesis: a Knowledge Synthesis Chapter. Ottawa, Ontario, Canada: Canadian Institutes of Health Research, 2010. (accessed 21 Apr 2017).

38. Higgins JP, Altman DG, Gøtzsche PC, et al. The Cochrane Collaboration's tool for assessing risk of bias in randomised trials. BMJ 2011;343:d5928. 